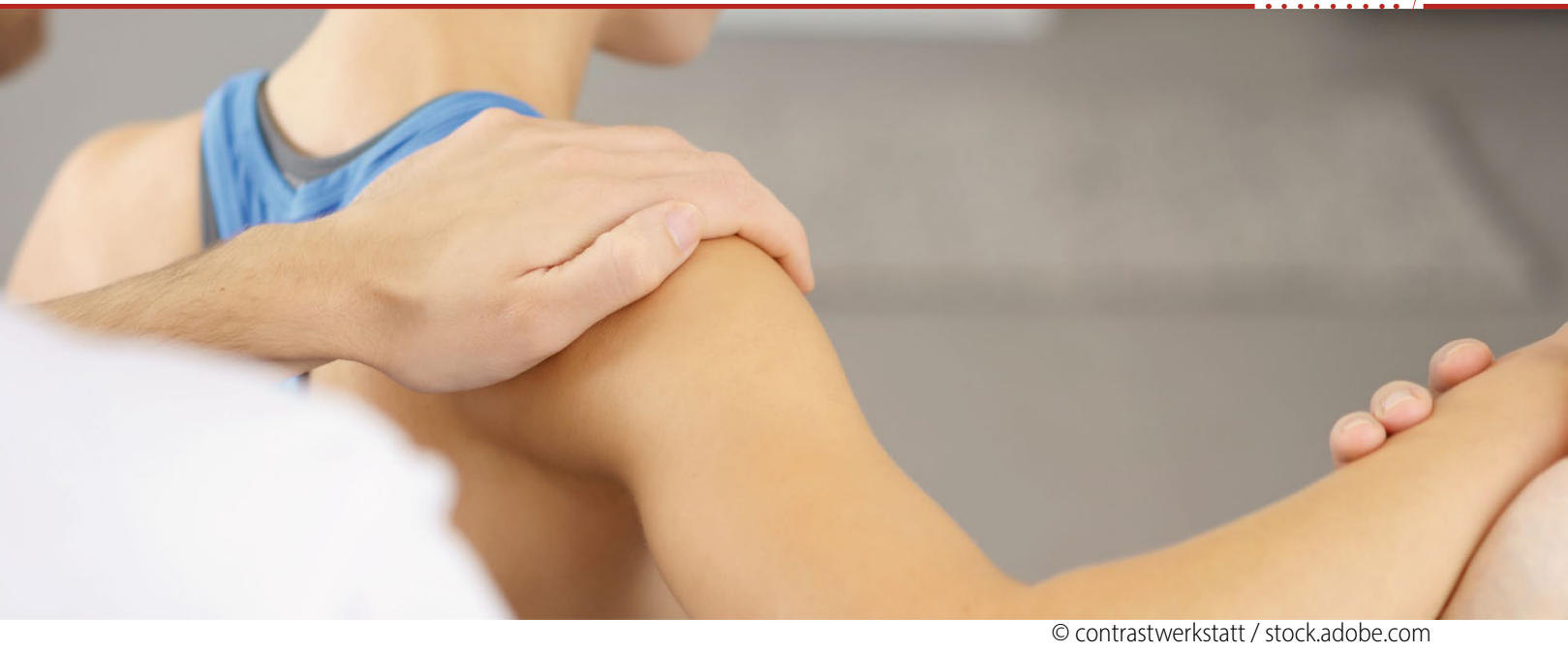

Siddhartha Popat M.A.

St. Katharinen, Deutschland

\title{
Schulterschmerzen
}

Der Themenschwerpunkt dieses Hefts ist der Schulterschmerz. In meiner Masterarbeit beim Studiengang Komplementärmedizin, Kulturwissenschaften und Heilkunde an der Europauniversität Viadrina 2013 habe ich mich für dieses Thema entschieden und vor allem die psychosomatischen Zusammenhänge ausgearbeitet [1]. In der Zusammenstellung dieses Schwerpunkts spiegelt sich das wider.

\section{I) Einen besonde-}

\section{ren Stellenwert nimmt die psychosomatische Betrachtung ein}

Die Bandbreite des Schwerpunkts geht von der gründlichen Untersuchung der Schulter aus (Dr. med. Rainer Wander) über die Behandlung mit Neuraltherapie (Dr. med. Udo Franke) und verschiedene Varianten der Akupunktur bis hin zur Schnittstelle von Naturheilkunde und Operation zu einer Betrachtung der Operationsindikationen (Dr. Harris Georgousis). Einen besonderen Stellenwert nimmt die psychosomatische Betrachtung ein, wobei aus unterschiedlichen Blickrichtungen die Kollegen Milz und Saha ihre Akzente setzen. Abgerundet wird der Schwerpunkt von der Darstellung der
Möglichkeiten mit Tuina durch Dr. Bachmann und einen wundervollen Fallbericht unseres Chefredakteurs Dr. Ots durch die Mikroakupunktur nach Prof. Yamamura aus Sao Paulo, die ihn selber nachdrücklich von seinen Schulterschmerzen befreit hat. Dieses spezielle Thema werden wir in der nächsten Ausgabe ausführlich darstellen.

Ich selber habe die Beschwerden der Schulter im Rahmen der Masterarbeit damals vorwiegend in einem Zusammenhang des unteren HWS-Syndroms dargestellt und in den Erklärungen von Rüdiger Dahlke und Louise Hay viele Angebote gefunden. Insbesondere ist mir die Arbeit von Prof. Porges über die Polyvagaltheorie sehr ans Herz gewachsen, in der die Bedeutung des myelinisierten Smart Vagus mit seinem Ursprung im Nucleus ambiguus hervorgehoben wird [3]. Diese entsendet eben auch sehr viel Fasern an den Nervus accessorius, welcher $u$. a. den M. trapezius und den M. sternocleidomastoideus innerviert.

In unserer sehr von Stress und überhöhten Ambitionen geprägten Zeit führt dieses natürlich zu einer häufigen Anund Verspannung der genannten Muskulatur und einem „Hochziehen“ der Schulter mit sämtlichen negativen statischen Folgen und negativen Einflüssen auf die Trophik der Muskulatur. Übersetzt man dieses in die Denkweise der chinesischen
Medizin, liegt natürlich eine typische Syndromdiagnose nahe, die Leberenergiestauung.

Körperlich führt die Anspannung dieser Muskulatur oft zu einer reflektorischen Anspannung in den Bereichen der ventralen Halsmuskulatur, also der Mm. scaleni und des M. pectoralis major und minor, und somit auch zu einem gestörten Lymphabfluss aus der oberen Extremität.

Alles dies kann im Rahmen der Behandlungssitzungen dem Patienten bewusst gemacht werden und damit immer wieder über die Zusammenhänge der Meridiane, Organzugehörigkeiten und Emotionen dem Patienten angeboten werden, sich den psychosomatischen Aspekten zu öffnen und diese zur Sprache zu bringen (Tab. 1). Häufig ist hierbei eine für den Patienten erleichternde emotionale Erkenntnis zu gewinnen, die in meiner Erfahrung einen Wendepunkt im Behandlungsverlauf ermöglicht.

In meiner Beobachtung ist es letztlich eigentlich sekundär, womit dann der Erfolg therapeutisch gesichert wird, sei es über die Segmentakupunktur (Dr. med. Reinhart Wagner), die Neuraltherapie, bioadaptive Impulse (Physiokey/ SCENAR), die Mikroakupunktursysteme (MAPS), Tuina oder manuelle Therapie (Dr. med. Jürgen Bachmann). Die Injektion von Ozon an die Gelenkkapsel oder sogar in das Gelenk, an die Bursa suba- 


\section{Tab. 1 Wirbelsäulenverschiebungen [2, S. 86]}

\begin{tabular}{l|l} 
Wirbel & Vermutliche Ursache \\
\hline C1 & $\begin{array}{l}\text { Angst, Verwirrung. Läuft vor dem Leben davon. Fühlt sich nicht } \\
\text { gut genug.,Was würden die Nachbarn dazu sagen?" Nicht enden } \\
\text { wollender innerer Dialog }\end{array}$ \\
\hline C2 & $\begin{array}{l}\text { Ablehnung von Weisheit. Weigerung, zu wissen oder zu verste- } \\
\text { hen. Unentschlossenheit. Groll und Vorwurf. Verhältnis zum Leben } \\
\text { unausgeglichen. Leugnung der eigenen Spiritualität }\end{array}$ \\
\hline C3 & $\begin{array}{l}\text { Nimmt Vorwürfe anderer auf sich. Schuldgefühle. Märtyrer. } \\
\text { Unentschlossenheit. Macht sich selbst nieder. Schluckt mehr, als } \\
\text { man verdauen kann }\end{array}$ \\
\hline C4 & $\begin{array}{l}\text { Schuldgefühle. Unterdrückte Wut. Verbitterung. Angstgestaute } \\
\text { Gefühle. Ungeweinte Tränen }\end{array}$ \\
\hline C5 & $\begin{array}{l}\text { Angst vor Lächerlichkeit und Demütigung. Angst sich selbst aus- } \\
\text { zudrücken. Lehnt das Gute in sich selbst ab. Überlastet }\end{array}$
\end{tabular}

\section{Neues Denkmuster}

Ich bin in meiner Mitte, ruhig und ausgeglichen. Das Universum stimmt mir bei. Ich vertraue meinem höheren Selbst. Alles ist gut

Ich bin eins mit dem Universum und allem Leben. Es ist gut für mich, zu wissen und zu wachsen

Ich bin nur für mich selbst verantwortlich und ich freue mich über mich selbst. Ich kann alles handhaben, was ich erschaffe

Ich bin klar in meiner Kommunikation mit dem Leben. Ich bin frei, das Leben gerade jetzt zu genießen

Meine Kommunikation ist klar. Ich nehme das Gute in mir an. Ich lasse ab von allen Erwartungen. Ich werde geliebt und bin in Sicherheit

cromialis oder die lange Bizepssehne hat ebenso häufig einen durchschlagenden Erfolg. Wenn aber die psychoemotionale Ebene negiert wird, bessern sich die Symptome des Patienten sehr oft nicht.

Ich wünsche bei der Lektüre dieses Schwerpunkts viele neue Erkenntnisse und freue mich auf Ihre Rückmeldungen an mich und die gesamte Redaktion!

\section{Korrespondenzadresse}

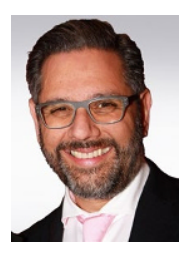

\section{Dr. med. Siddhartha}

Popat M.A.

Kolpingstraße 8, 53562 St.

Katharinen, Deutschland

sidpopat@aol.com

Interessenkonflikt. S. Popat gibt an, dass kein Interessenkonflikt besteht.

Literatur

1. Siddhartha Popat (2013) Die psychoemotionalen Aspekte der Behandlung von chronischen Schmerzen am Beispiel des unteren HWS Syndroms. Masterarbeit. Europauniversität Viadrina, Frankfurt (Oder)

2. Hay L (1989) Heile deinen Körper. Seelisch-geistige Gründe für körperliche Krankheit. Lüchow Verlag, Bielefeld

3. Porges SW (2010) Die Polyvagal-Theorie: Neurophysiologische Grundlagen der Therapie. Emotionen, Bindung, Kommunikation \& ihre Entstehung, 2. Auff. Junfermann Verlag, Paderborn 